\title{
Pharmacokinetic Modeling of Non-Linear Brain Distribution of Fluvoxamine in the Rat
}

\author{
Marian Geldof, ${ }^{1,2}$ Jan Freijer, ${ }^{3,4}$ Ludy van Beijsterveldt, ${ }^{5}$ and Meindert Danhof ${ }^{1,3,6}$
}

Received January 2, 2007; accepted June 25, 2007; published online August 21, 2007

Introduction. A pharmacokinetic (PK) model is proposed for estimation of total and free brain concentrations of fluvoxamine.

Materials and methods. Rats with arterial and venous cannulas and a microdialysis probe in the frontal cortex received intravenous infusions of $1,3.7$ or $7.3 \mathrm{mg} \cdot \mathrm{kg}^{-1}$ of fluvoxamine.

Analysis. With increasing dose a disproportional increase in brain concentrations was observed. The kinetics of brain distribution was estimated by simultaneous analysis of plasma, free brain ECF and total brain tissue concentrations. The PK model consists of three compartments for fluvoxamine concentrations in plasma in combination with a catenary two compartment model for distribution into the brain. In this catenary model, the mass exchange between a shallow perfusion-limited and a deep brain compartment is described by a passive diffusion term and a saturable active efflux term.

Results. The model resulted in precise estimates of the parameters describing passive influx into $\left(k_{\text {in }}\right)$ of $0.16 \mathrm{~min}^{-1}$ and efflux from the shallow brain compartment $\left(k_{\text {out }}\right)$ of $0.019 \mathrm{~min}^{-1}$ and the fluvoxamine concentration at which $50 \%$ of the maximum active efflux $\left(C_{50}\right)$ is reached of $710 \mathrm{ng} \cdot \mathrm{ml}^{-1}$. The proposed brain distribution model constitutes a basis for precise characterization of the PK-PD correlation of fluvoxamine by taking into account the non-linearity in brain distribution.

KEY WORDS: blood-brain barrier; intracerebral microdialysis; PK-PD correlation; selective serotonin re-uptake inhibitor; transporter.

\section{INTRODUCTION}

Selective Serotonin Reuptake Inhibitors (SSRIs) are the first-line treatment for depression $(1,2)$. SSRIs selec-

\footnotetext{
${ }^{1}$ Division of Pharmacology, Leiden-Amsterdam Center for Drug Research, Leiden University, P.O. Box 9502, 2300 RA, Leiden, The Netherlands.

${ }^{2}$ Present address: Department of Drug Metabolism and Pharmacokinetics, Janssen Pharmaceutica N.V., Johnson and Johnson Pharmaceutical Research and Development, Turnhoutseweg 30, 2340, Beerse, Belgium.

${ }^{3}$ LAP \&P Consultants BV, Archimedesweg 31, 2333 CM, Leiden, The Netherlands.

${ }^{4}$ Present address: Astellas Pharma, Europe R\&D, P.O. Box 108, 2350 AC, Leiderdorp, The Netherlands.

${ }^{5}$ Department of Clinical Pharmacology, Janssen Pharmaceutica N.V., Johnson Pharmaceutical Research and Development, Turnhoutseweg 30, 2340, Beerse, Belgium.

${ }^{6}$ To whom correspondence should be addressed. (e-mail: m.danhof@ lacdr.leidenuniv.nl)
}

ABBREVIATIONS: 5-HT, 5-hydroxytryptamine; $A_{50}$, fluvoxamine amount in deep brain compartment at which $50 \%$ of saturation of the active removal flux is reached; $A_{\mathrm{DB}}$, amount of fluvoxamine in deep brain compartment; AP, Anterior-Posterior; $A_{\mathrm{SP}}$, amount of fluvoxamine in shallow perfusion-limited compartment; $A_{\mathrm{T}}$, total amount of fluvoxamine in the brain; BBB, blood-brain barrier; BSA, Bovine Serum Albumin; $C_{50}$, fluvoxamine concentration in deep brain compartment at which $50 \%$ of saturation of the active removal flux is reached; $C_{\mathrm{B}}$, total fluvoxamine concentration in the brain.; $C_{\mathrm{DB}}$, fluvoxamine concentration in deep brain compartment; tively and powerfully block the serotonin transporter (SERT) and thereby the reuptake of serotonin (5-HT) in the presynaptic nerve terminal yielding increased extracellular 5-HT levels and enhancement of serotonergic neuro-

$C_{\text {in }}$, fluvoxamine concentration entering the brain; $C_{\text {in-rec }}$, fluvoxamine concentration entering microdialysis probe; CL, systemic clearance; $C_{\text {out }}$, fluvoxamine concentration leaving the brain; $C_{\text {out-rec }}$, fluvoxamine concentration leaving microdialysis probe; $C_{\mathrm{p}}$, fluvoxamine plasma concentration; CSF, cerebrospinal fluid; $C_{\mathrm{SP}}$, fluvoxamine concentration in shallow perfusion-limited compartment; $C_{\mathrm{T}}$, total fluvoxamine concentration in the brain; C.V., Coefficient of Variation; DMSO, dimethylsulfoxide; ECF, extracellular fluid; $f_{\mathrm{DB}}$, partition coefficient for deep brain compartment; $f_{\mathrm{SP}}$, partition coefficient for shallow perfusion-limited compartment; IIV, inter-individual variability; $k_{\text {diff }}$, diffusion rate constant; $k_{\text {in }}$, influx rate constant in the brain; $k_{\text {out }}$, efflux rate constant from the brain; L, Lateral; LC-MS/MS, liquid chromatographymass spectrometry/mass spectrometry; MRP, Multidrug Resistance Protein; MVOF, Minimum Value of Objective Function; $\mathrm{NaOH}$, sodium hydroxide; $N_{\max }$, maximal active removal flux; NONMEM, nonlinear mixed effects modeling; $N_{\text {SP-DB }}$, net mass exchange between shallow perfusion-limited compartment and deep brain compartment; $P$, partition coefficient; PD, pharmacodynamics; Pgp, P-glycoprotein; PK, pharmacokinetics; PVP, polyvinylpyrrolidone; $Q 2, Q 3$, intercompartmental clearances; $Q_{\mathrm{B}}$, effective plasma perfusion rate;

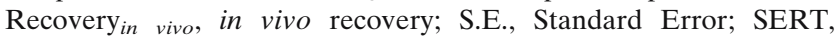
serotonin transporter; SSRI, Selective Serotonin Re-uptake Inhibitor; $V$, Vertical; $V 1$, central volume of distribution; $V 2, V 3$, peripheral volumes of distribution; $V_{\mathrm{T}}$, brain volume; $V_{\mathrm{DB}}$, volume in deep brain compartment; $V_{\mathrm{SP}}$, volume in shallow perfusion-limited compartment. 
transmission $(3,4)$. Despite their widespread use and numerous pre-clinical and clinical investigations, very few studies have addressed the pharmacokinetic-pharmacodynamic (PKPD) correlations of SSRIs. This is remarkable since the PKPD correlations of SSRIs appear to be rather complex. Upon chronic treatment important time dependencies in the pharmacodynamics of SSRIs have been observed (5-7).

In recent years, important progress has been made in the field of mechanism-based PK-PD modeling. The objective of mechanism-based PK-PD modeling is to understand, in a strictly quantitative manner, the mechanisms which determine the time-course of the intensity of the drug effect in vivo. A pertinent feature of mechanism-based PK-PD models is that they contain specific expressions to describe processes on the causal path between drug administration and response. This includes the target site distribution, the target site binding, the target site activation and the homeostatic feedback mechanisms (8-12).

Modeling of target site distribution kinetics is particularly important for drugs which act in tissues that are protected by specific barriers such as the central nervous system $(8,9)$. In recent years important progress has been made in elucidating the functionality of the blood-brain barrier and the mechanisms of drug transport to the brain It has been demonstrated that the distribution of drugs into the brain is restricted for hydrophilic drugs and for compounds which are substrates for efflux transporters at the blood-brain barrier (13). Over the years also various pharmacokinetic brain distribution models have been proposed (14), which in theory constitute a scientific basis for the description of target site distribution kinetics in mechanism-based PK-PD modeling. To date however, nonlinearity in brain distribution has not been taken into account.

The present investigation focuses on the pharmacokinetic modeling of the brain distribution of fluvoxamine. Recently, evidence has been obtained that fluvoxamine may be a substrate for active efflux transporters at the blood-brain barrier. Specifically, it has been shown that fluvoxamine inhibits Pgp in MDR1 cells (a model for human Pgp) as well as in primary porcine brain capillary endothelial cells (model for the blood-brain barrier) (15). On the other hand, no significant Pgp mediated fluvoxamine efflux in MDR-1 cells was observed in another investigation (16).

The objective of the present investigation was to characterize in a strictly quantitative manner the kinetics of the brain distribution of fluvoxamine in rats in vivo, including any pertinent non-linearities To this end, the time-course of the fluvoxamine concentration in plasma, brain microdialysate and brain tissue was determined following intravenous administration of pharmacologically relevant doses of $1,3.7$ or 7.3 $\mathrm{mg} / \mathrm{kg}$. On the basis of these results a PK model is proposed which enables precise estimation of the time course of both the total and the free fluvoxamine concentration in the brain.

\section{PHARMACOKINETIC MODEL FOR FLUVOXAMINE BRAIN DISTRIBUTION}

The proposed model for the brain distribution kinetics of fluvoxamine is an extension of a model proposed earlier for the brain distribution of thiopental $(14,17)$ and is illustrated in Fig. 1. The brain distribution model consists of three compartments to describe the concentration versus time profile in

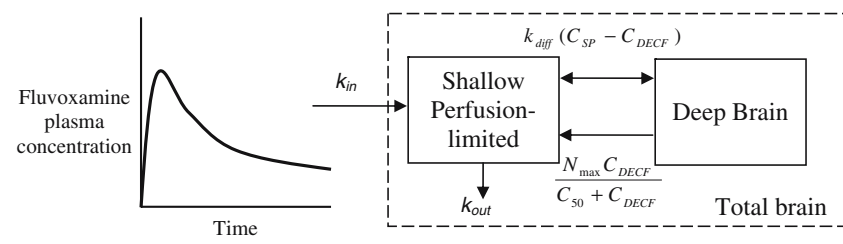

Fig. 1. Schematic representation of the proposed pharmacokinetic model for fluvoxamine in brain. The fluvoxamine plasma concentration versus time profiles were predicted by a previously developed population PK model and served as input function for fluvoxamine in the brain. The catenary brain distribution model consists of a shallow perfusion-limited brain compartment and a deep brain compartment. The mass exchange of fluvoxamine between these compartments is composed of a passive diffusion term and an saturable active removal flux $\left(k_{10}=\right.$ elimination rate constant from central plasma compartment; $k_{\text {in }}=$ rate constant for distribution into the shallow brain compartment; $k_{\text {out }}=$ rate constant for distribution from the shallow brain compartment; $k_{\text {diff }}=$ diffusion rate constant between the shallow perfusion-limited and the deep brain compartment; $C_{\mathrm{SP}}=$ concentration in shallow perfusion-limited compartment; $C_{\mathrm{DB}}=$ concentration in deep brain compartment; $N_{\max }=$ maximal active removal flux; $C_{50}=$ fluvoxamine concentration in the deep brain compartment at which $50 \%$ of saturation of the active removal flux is reached).

plasma in combination with a catenary two compartment model to describe the distribution of fluvoxamine into the brain. Within the catenary model, the first brain compartment is a shallow compartment that is in direct contact with the blood flow and where the concentration is determined by perfusion (shallow perfusion-limited compartment). The second compartment is a deep brain compartment in which the concentration is equal to the measured ECF concentrations (deep brain compartment). Fluvoxamine is not able to enter the deep brain compartment directly, but only indirectly from the shallow perfusion-limited compartment. The transport between the two brain compartments is by diffusion and/or active transport (influx and/or efflux) mediated by P-glycoprotein (Pgp) and/or other transporters. Therefore, the distribution of fluvoxamine into the brain is determined by perfusion of the outer shallow brain tissues (subscript SP) and exchange with the deep brain compartment (subscript DB):

$$
\begin{gathered}
\frac{d A_{s p}}{d t}=Q_{B} C_{\text {in }}-Q_{B} C_{\text {out }}+N_{S P-D B} \\
\frac{d A_{D B}}{d t}=-N_{S P-D B}
\end{gathered}
$$

in which $A_{S P}$ is the amount of fluvoxamine in the shallow perfusion-limited compartment, $Q_{B}$ is the effective plasma perfusion rate, $C_{i n}$ is the concentration entering the shallow perfusion-limited compartment, $C_{\text {out }}$ is the concentration leaving the shallow perfusion-limited compartment, $N_{S P-D B}$ is the net mass exchange between the shallow perfusionlimited compartment and deep brain compartment and $A_{D B}$ is the amount of fluvoxamine in the deep brain compartment.

The mass exchange of fluvoxamine between the outer perfusion-limited brain compartment and the deep brain compartment contains two components, a passive diffusion term 
and an active removal flux. By assuming that this active removal flux is saturable the following relation for $N_{S P-D B}$ is obtained:

$$
N_{S P-D B}=-k_{\text {diff }}\left(C_{S P}-C_{D B}\right)+N_{\max } C_{D B} /\left(C_{50}+C_{D B}\right)
$$

in which $k_{\text {diff }}$ is the diffusion rate constant between the shallow perfusion-limited and deep brain compartment, $C_{S P}$ is the concentration in the shallow perfusion-limited compartment, $C_{D B}$ is the concentration in the deep brain compartment, $N_{\max }$ is the maximal active removal flux and $C_{50}$ is the fluvoxamine concentration in the deep brain compartment at which $50 \%$ of saturation of the active removal flux is reached.

In the model the values of the rate constants for diffusion from the shallow perfusion-limited brain compartment and the deep brain compartment and vice versa were assumed to be identical. Moreover, in the data-analysis the value of the parameter $k_{\text {diff }}$ rose to high values, allowing further reduction of the model by elimination of this parameter. Thus ultimately rapid equilibrium between fluvoxamine concentrations in the shallow perfusion-limited and the deep brain compartment was assumed resulting in the following relationship between the concentrations in the shallow perfusion-limited compartment and the deep brain compartment:

$$
C_{S P}=C_{D B}+\frac{N_{\max }}{K_{\text {diff }}} C_{D B} /\left(C_{50}+C_{D B}\right)
$$

The total amount of fluvoxamine in the brain can be described by the following equation:

$$
\frac{d A_{T}}{d t}=\frac{d A_{S P}}{d t}+\frac{d A_{D B}}{d t}=Q_{B} C_{\text {in }}-Q_{B} C_{\text {out }}
$$

in which $A_{T}$ is the total amount of fluvoxamine in the brain.

The concentration entering the brain $\left(C_{i n}\right)$ is assumed to be equal to the plasma concentration:

$$
C_{\text {in }}=C_{P}
$$

in which $C_{p}$ is the plasma concentration.

The concentration leaving the brain $\left(C_{\text {out }}\right)$ is determined by the partition coefficient $(P)$ between drug in plasma and the concentration in the shallow perfusion-limited brain area:

$$
C_{\text {out }}=C_{S P} / P
$$

In addition, the following constants can be defined:

$$
\begin{gathered}
k_{\text {in }}=Q_{B} / V_{T} \\
k_{\text {out }}=Q_{B} /\left(V_{T} P\right)
\end{gathered}
$$

in which $k_{\text {in }}$ is the influx rate constant in the brain, $V_{T}$ is the volume of the brain and $k_{\text {out }}$ is the efflux rate constant from the brain.

The differential equation for total fluvoxamine concentration in the brain can be described by:

$$
\frac{d C_{T}}{d t}=k_{\text {in }} C_{p}-k_{\text {out }} C_{S P}
$$

in which $C_{T}$ is the total fluvoxamine concentration in the brain.
The relationships between the concentration in the deep brain compartment and total brain and the concentration in the shallow perfusion-limited brain compartment and total brain are defined by:

$$
\begin{gathered}
C_{D B}=f_{D B} C_{T} \\
C_{S P}=f_{S P} C_{T}
\end{gathered}
$$

in which $f_{D B}$ and $f_{S P}$ are partition coefficients for the deep brain compartment and shallow perfusion-limited compartment, respectively.

The partition coefficient for the deep brain compartment can be calculated by the following formula:

$$
\begin{aligned}
& f_{D B}= \\
& \frac{\left.-N * * *_{\max }+C_{T}-C_{50}+\sqrt{\left(N_{* * *} \max \right.}-C_{T}+C_{50}\right)^{2}+4 C_{T} C_{50}}{2 C_{T}}
\end{aligned}
$$

in which $N_{* * *} \max$ is a lumped parameter that can be defined by:

$$
N_{* * *} \max =\frac{N_{\max } V_{S P}}{k_{\text {diff }} V_{D B} \alpha}>0
$$

with $\alpha$ defined by:

$$
\alpha=\left(\frac{V_{S P}}{V_{D B}}+1\right)>1
$$

Furthermore, the partition coefficient for the shallow perfusion-limited compartment can be calculated by the following equation:

$$
f_{S P}=1+V_{D B} / V_{S P}\left(1-f_{D B}\right)
$$

The complete mathematical derivations of the model are presented in Appendix.

The schematic representation of the proposed PK model in Fig. 1 shows that at low fluvoxamine plasma concentrations relatively low fluvoxamine concentrations will be present in the deep brain compartment because of the action of the active efflux transport at the interface of the shallow and the deep brain compartment. At higher fluvoxamine concentrations, this active efflux transport becomes saturated and therefore relatively higher concentrations of fluvoxamine are observed in the deep brain compartment.

\section{MATERIALS AND METHODS}

\section{Chemicals}

Fluvoxamine maleate and clovoxamine fumarate were kindly provided by Solvay Pharmaceuticals (Weesp, The Netherlands). Dimethylsulfoxide (DMSO), sodium hydroxide $(\mathrm{NaOH})$ and isoamylalcohol were purchased from Merck (Darmstadt, Germany). Isopentane, ethanol, acetonitrile and methanol were obtained from Acros (Geel, Belgium). Heptane was purchased from Sigma-Aldrich Laborchemikalien (Seelze, Germany) and ammonium acetate was obtained from Baker Chemicals (Deventer, The Netherlands). Millipore water was obtained from a Milli-Q system (Millipore SA, Molsheim, France). 


\section{Animals}

Male Wistar rats (Charles River Wiga GMBH, Sulzfeld, Germany) weighing 226-250 g were housed in groups for 6-10 days, under standard environmental conditions (ambient temperature $21^{\circ} \mathrm{C}$, humidity $60 \%, 12$-h light/dark cycle). The animals had free access to food (laboratory chow, Hope Farms, Woerden, The Netherlands) and acidified water. For the microdialysis studies 26 animals were used and for the brain sampling studies 35 animals were used. After surgery (i.e. for the implantation of arterial and venous cannulas and, if applicable, a dummy microdialysis probe), the animals in the microdialysis studies were housed individually for 1 week and the animals in the brain sampling studies for 2 days. The study protocol was approved by the Ethical Committee on Animal Experimentation of Leiden University.

\section{EXPERIMENTAL PROCEDURES}

\section{Microdialysis Studies}

\section{Surgical Procedures}

Animals were anaesthetized by a subcutaneous injection of $0.1 \mathrm{ml} / 100 \mathrm{~g}$ Ketanest-S ${ }^{\circledR}((S)$-ketaminebase, Parke-Davis, Hoofddorp, The Netherlands) and an intramuscular injection of $0.01 \mathrm{ml} / 100 \mathrm{~g}$ Domitor ${ }^{\circledR}$ (medetomidine hydrochloride, Pfizer, Capelle a/d IJssel, The Netherlands). A cannula was implanted in the right jugular vein $(10 \mathrm{~cm}$ polyethylene tubing, Portex Limited, Hythe, United Kingdom; I.D.=0.58, O.D. $=0.96 \mathrm{~mm}$ ) for fluvoxamine administration and a cannula in the left femoral artery (4 cm polyethylene tubing, Portex Limited; I.D. $=0.28$, O.D. $=0.61 \mathrm{~mm}$ heat-sealed to 18 $\mathrm{cm}$ polyethylene tubing, I.D.=0.58, O.D. $=0.96 \mathrm{~mm}$ ) for collection of blood samples. The cannulas were subcutaneously tunneled and externalized at the back of the neck. The venous cannula was filled with saline $(0.9 \%$, B. Braun Melsungen AG, Melsungen, Germany) containing heparin (20 IU.ml ${ }^{-1}$, Pharmacy, Leiden University Medical Center, Leiden, The Netherlands) and the arterial cannula was filled with $25 \%(w / v)$ polyvinylpyrrolidone (PVP, Brocacef, Maarssen, The Netherlands) solution in saline $(0.9 \%)$ containing heparin $\left(20 \mathrm{IU} \cdot \mathrm{ml}^{-1}\right)$ to prevent blockade by blood clotting. After implantation of the cannulas, the animals were instrumented with a microdialysis guide cannula (CMA/12, Aurora Borealis Control BV, Schoonebeek, The Netherlands) within the same surgery. A microdialysis guide cannula with a dummy probe was implanted in the frontal cortex (AP: 3.2; L: -3.0 ; V: $-1.5 \mathrm{~mm}$ from bregma, in accordance with the atlas of Paxinos and Watson (18)). Two support screws were placed and the guide was secured in place using dental cement (Simplex Rapid liquid and powder, Kemdent Associated Dental Products, United Kingdom).

\section{Microdialysis}

One day before implantation of the microdialysis probe, the probes (CMA/12, membrane length $3 \mathrm{~mm}$, Aurora Borealis Control BV) were perfused ex vivo overnight with artificial cerebrospinal fluid (containing: $145 \mathrm{mM} \mathrm{NaCl}, 0.6$ $\mathrm{mM} \mathrm{KCl}, 1.0 \mathrm{mM} \mathrm{MgCl}, 1.2 \mathrm{mM} \mathrm{CaCl}_{2}, 0.2 \mathrm{mM}$ ascorbic acid in $2 \mathrm{mM}$ phosphate buffer $\mathrm{pH}$ 7.4) (19). The dummy probe was replaced by the microdialysis probe 1 day before start of the study. Because fluvoxamine appeared to be adhesive to the microdialysate tubing and microdialysis probe, the artificial cerebrospinal fluid additionally contained $0.5 \%(w / v)$ Bovine Serum Albumin (BSA, Sigma-Aldrich, Zwijndrecht, The Netherlands), which completely dissolved the adhesion problems (data not shown). Flow rate during the whole experiment was constant at $2 \mu \mathrm{l} \cdot \mathrm{min}^{-1}$ and the outlet tubing was connected to a microdialysate fraction collector (Univentor 820, Antec Leyden BV, Zoeterwoude, The Netherlands). After intravenous administration of fluvoxamine, dialysate concentrations were measured over a period of $5 \mathrm{~h}$. Sampling intervals were 20 or 30 min yielding a volume of 40 or $60 \mu$ l fluvoxamine dialysate sample in both microdialysis studies. At the end of the experiments, brains of the animals were removed and the location of the microdialysis probe was verified by visual inspection. Microdialysate samples were collected at a temperature of $4^{\circ} \mathrm{C}$ in the fraction collector, and subsequently stored at $-20^{\circ} \mathrm{C}$ until analysis. Dosages and observed fluvoxamine concentrations are expressed as free base.

\section{In Vivo Recovery}

For 20 of the 26 animals used in the microdialysis studies, individual values for the in vivo recovery were determined by the method reverse dialysis or retrodialysis by drug after $1 \mathrm{~h}$ of stabilization (20,21). For the remaining six animals, the mean in vivo recovery value was used to determine true unbound fluvoxamine concentrations in ECF from dialysate concentrations. To exclude any non-linearity in the in vivo recovery, in the retrodialysis experiments, a range of fluvoxamine concentrations $\left(6,12,30\right.$ and $\left.60 \mathrm{ng} \cdot \mathrm{ml}^{-1}\right)$ in artificial cerebrospinal fluid containing $0.5 \%$ BSA were locally infused in the frontal cortex. Six dialysate fractions of $20 \mathrm{~min}$ were collected, of which the median four fractions were used for determination of the in vivo recovery value. This in vivo recovery was calculated per probe by the loss of fluvoxamine from the fluvoxamine retrodialysis solution to the surrounding tissue according to the following equation:

$$
\text { Recovery }_{\text {invivo }}=\frac{\left(C_{\text {in-rec }}-C_{\text {out }- \text { rec }}\right)}{C_{\text {in-rec }}}
$$

in which $C_{\text {in-rec }}$ is the fluvoxamine concentration entering the probe and $C_{\text {out-rec }}$ is the fluvoxamine concentration leaving the probe. At the end of the retrodialysis phase, the probes were perfused for $2 \mathrm{~h}$ with artificial cerebrospinal fluid containing $0.5 \%$ BSA after which no fluvoxamine was detected in dialysate samples and the administration of fluvoxamine in the jugular vein could be started.

\section{Dosage Regimen}

From the 26 animals used in the microdialysis studies, 8 animals received $1 \mathrm{mg} . \mathrm{kg}^{-1}, 8$ animals received $3.7 \mathrm{mg} . \mathrm{kg}^{-1}$ and 10 animals received $7.3 \mathrm{mg} \cdot \mathrm{kg}^{-1}$ fluvoxamine via a 30-min intravenous infusion in the jugular vein cannula at a flow rate of $20 \mu \mathrm{l} . \mathrm{min}^{-1}$ using a BAS BeeHive pump (Bioanalytical Systems, West Lafayette, USA). 


\section{Blood Sampling}

A number of 13 arterial blood samples $(100 \mu \mathrm{l})$ were collected at variable fixed time intervals from the cannula in the femoral artery over a time period between 0 and $5 \mathrm{~h}$ after fluvoxamine administration. After collection of each blood sample, an equal volume of heparinized $0.9 \% \mathrm{NaCl}(20$ IU.ml ${ }^{-1}$ ) was administered to the animal. Blood samples were collected in heparinized eppendorf tubes and kept on ice during the experiment. After centrifugation $(10 \mathrm{~min}$, $5,000 \times g), 50 \mu \mathrm{l}$ plasma was transferred into a glass tube and stored at $-20^{\circ} \mathrm{C}$ until sample analysis.

\section{Brain Sampling Studies}

\section{Surgical Procedures}

Animals in the brain sampling studies were implanted with a cannula in the right jugular vein for fluvoxamine administration and a cannula in the left femoral artery for collection of blood samples as described for the animals in the microdialysis studies.

\section{Dosage Regimen}

From the 35 animals used in the brain sampling studies, 19 animals received $1 \mathrm{mg} . \mathrm{kg}^{-1}$ and 16 animals received 7.3 $\mathrm{mg} . \mathrm{kg}^{-1}$ fluvoxamine via the 30 -min intravenous infusion in the jugular vein cannula as described for the microdialysis studies.

\section{Blood Sampling}

A number between 2 and 15 arterial blood samples (100 $\mu \mathrm{l})$ were collected from the animals between 2.5 and $600 \mathrm{~min}$ from the cannula in the femoral artery after fluvoxamine administration, dependent on the time of brain sampling. Blood samples were collected, handled and stored as described for the microdialysis studies.

\section{Brain Collection}

From the 19 animals that received $1 \mathrm{mg} \cdot \mathrm{kg}^{-1}$ fluvoxamine, the brains were collected at 10, 20, 30, 45, 75, 90, 105, 120, $135,150,180,210,240,270,300,330,360,390$ and $420 \mathrm{~min}$ after fluvoxamine administration. From the 16 animals that received $7.3 \mathrm{mg} \cdot \mathrm{kg}^{-1}$ fluvoxamine, the brains were collected at $15,30,45,60,75,90,105,120,135,200,250,550,600,650$, 700 and $750 \mathrm{~min}$ after fluvoxamine administration. The animals were sacrificed by decapitation after which the brains were quickly isolated. The brains were frozen in dry ice-cooled isopentane that was surrounded with cold ethanol and stored at $-20^{\circ} \mathrm{C}$ until analysis of fluvoxamine brain levels.

\section{Drug Analysis}

Fluvoxamine samples in the microdialysis studies and brain sampling studies were analyzed for fluvoxamine using liquid chromatography with tandem mass spectrometry (LC-MS/MS) as has been described earlier for fluvoxamine plasma (22) and for fluvoxamine in ECF and brain. Calibration standards and independent quality control samples were prepared by addition of fluvoxamine solutions in DMSO to control plasma, artificial cerebrospinal fluid or brain. Briefly, in plasma samples, proteins were precipitated by addition of acetonitrile and a volume of $20 \mu \mathrm{l}$ of supernatant was injected into the system. For the ECF samples, a volume of $50 \mu \mathrm{l}$ of the calibration standards or QC samples in DMSO were added to $50 \mu$ l artificial cerebrospinal fluid. For the ECF samples from the rats, a volume of $50 \mu \mathrm{l}$ DMSO, $500 \mu \mathrm{l} \mathrm{H}_{2} \mathrm{O}$ and $50 \mu \mathrm{l}$ of $500 \mathrm{ng} / \mathrm{ml}$ clovoxamine in DMSO were added. Addition of $100 \mu \mathrm{NaOH}$ increased the $\mathrm{pH}$ value to approximately 12. A volume of $4 \mathrm{ml}$ of heptane-isoamylalcohol $(95: 5, v / v)$ was added, the solution was centrifuged $(10 \mathrm{~min}, 3000 \times \mathrm{g})$ and the organic layer was evaporated to dryness under nitrogen at $65^{\circ} \mathrm{C}$. The residues were dissolved in a mixture of $10 \mathrm{mM}$ ammonium acetate and acetonitrile $(50: 50, v / v)$ and a volume of $30 \mu \mathrm{l}$ was injected into the system. Control brains and brains from fluvoxamine treated rats were weighed and nine volumes of $\mathrm{H}_{2} \mathrm{O}$ were added before homogenization. A volume of 100 $\mu \mathrm{l}$ of the calibration standards or QC samples in DMSO were added to $1 \mathrm{ml}$ control brain homogenate. For the brain samples of the rats, a volume of $100 \mu \mathrm{l}$ DMSO and $100 \mu \mathrm{l}$ of $500 \mathrm{ng} / \mathrm{ml}$ clovoxamine in DMSO were added to $1 \mathrm{ml}$ of brain homogenate. A volume of $2 \mathrm{ml}$ of methanol was added for extraction and the samples were rotated for 15 min and centrifuged $(10 \mathrm{~min}, 5,000 \times g)$. The solutions were transferred in eppendorf tubes, centrifuged for another time (10 min, 9,727 $\times g$ ) and a volume of $20 \mu \mathrm{l}$ was injected into the system. All fluvoxamine samples were quantified on a reversed phase LC column (BDS Hypersil C18, $3 \mu \mathrm{m}$ particle size, $100 \times 4.6$ mm I.D.; Thermo Hypersil-Keystone, Brussels, Belgium). LC-MS/MS analysis was performed on an API-4000 MS/MS (Applied Biosystems, Toronto, Canada), coupled to an HPLC system (Agilent, Palo Alto, USA). The MS/MS operated in the positive ion mode using the TurbolonSpray-interface (electrospray ionization) was optimized for the quantification of fluvoxamine. For analysis of brain tissue samples, an additional guard cartridge (Hypersil ODS $5 \mu \mathrm{m}$ $10 \times 4.0 \mathrm{~mm}$ drop-in cartridge, Thermo Electron Corporation, Brussels, Belgium) with holder (Uniguard holder, Thermo Electron Corporation) was used. The intra-batch accuracy from independent QC samples was between 80 and $120 \%$ over the entire range of the samples. The limit of quantification for fluvoxamine was $1 \mathrm{ng} \cdot \mathrm{ml}^{-1}$ in plasma, brain ECF and brain tissue.

\section{Data Analysis}

A nonlinear mixed effects modeling approach was used to describe the concentration-time profiles of fluvoxamine in plasma, brain ECF and brain tissue from all individual animals simultaneously. All fitting procedures were performed on a personal computer (Intel ${ }^{\circledR}$ Pentium ${ }^{\circledR} 4$ processor) running under Windows XP using the Compaq Visual FORTRAN standard edition 6.1 (Compaq Computer Cooperation, Euston, Texas, USA) with the nonlinear mixed effects modeling software NONMEM (Version V, Level 1.1., NONMEM project group, University of California, San Francisco, USA). 


\section{PK Analysis in Plasma}

As described in the development of the fluvoxamine brain distribution model, the PK information in plasma obtained in the present study was analyzed in combination with plasma concentration time data of other studies on the basis of a previously proposed population three-compartment PK model for fluvoxamine in plasma (22). The individual plasma concentration versus time profiles in the animals included in the present study were estimated on the basis of the obtained post hoc estimates of the parameters and used as fluvoxamine input function in the brain. In Table I, the mean post hoc estimates for the PK parameters of fluvoxamine in plasma obtained by the three-compartment PK model are depicted. Included are the post hoc estimates for systemic clearance $(C L)$, central volume of distribution (V1), two peripheral volumes of distribution $(V 2, V 3)$ and inter-compartmental clearances $(Q 2, Q 3)$. On the basis of a covariate analysis, no differences in the pharmacokinetics of the three different dose groups nor between the rats in the microdialysis study and the brain sampling study could be detected.

\section{PK Analysis in Brain ECF and Brain Tissue}

All fluvoxamine data in plasma, brain ECF and brain tissue were implemented in the PREDD subroutine ADVAN 6 TOL5, which is a general nonlinear model that uses the numerical solution of the differential equations.

Inter-individual variability on the parameters was modeled by an exponential equation:

$$
P_{i}=\theta \cdot \exp \left(\eta_{i}\right)
$$

in which $P_{i}$ is the individual estimate for parameter $P$ for the $i$ th individual, $\theta$ is the population estimate for parameter $P$ and $\eta_{i}$ is the inter-individual random deviation of $P_{i}$ from $P$. The values of $\eta_{i}$ are assumed to be normally distributed with mean zero and variance $\omega^{2}$ that distinguished the pharmacokinetic parameters for the $i$ th individual from the population typical value $\theta$. Inter-individual variabilities were analyzed on each parameter and the inter-individual effects that did not significantly improve the model or could not be estimated were fixed to zero. Correlations between the inter-individual variabilities of the various parameters were explored using the OMEGA BLOCK option. Unexplained variability (e.g. caused by measurement and experimental errors) in fluvoxamine concentrations in ECF and total brain were best described by a proportional error:

$$
C m_{i j}=C_{i j} \cdot\left(1+\varepsilon_{i j}\right)
$$

in which $C m_{i j}$ is the measured ECF or brain concentration, $C_{i j}$ is the $j$ th ECF or brain concentration for the $i$ th individual predicted by the model and $\varepsilon_{i j}$ account for the residual deviance of the predicted from the observed concentration. The values for $\varepsilon$ were assumed to be independently normally distributed with mean zero and variance $\sigma^{2}$. Population pharmacokinetic values of $\theta, \omega^{2}$ and $\sigma^{2}$ were estimated using the first-order conditional estimation with interaction method in NONMEM. Model fits were compared on the basis of the likelihood ratio test $(23,24)$, diagnostic plots, parameter correlations and precision in parameter estimates. An additional parameter was included in the structural model if the resulting change in minimum value of the objective function (MVOF) was $\geq 6.6(p \leq 0.01)$.

\section{RESULTS}

\section{In Vivo Recovery of Microdialysis}

The average values of the recoveries were $0.20 \pm 0.09$, $0.36 \pm 0.10,0.27 \pm 0.15,0.25 \pm 0.07$ for retrodialysis fluvoxamine solutions of $6,12,30$ and $60 \mathrm{ng} \cdot \mathrm{ml}^{-1}$, respectively. Therefore, a mean recovery value of $0.27 \pm 0.10$ was used for the six animals in which no individual values of the in vivo recovery were obtained.

\section{PK Analysis in Plasma}

By application of the previously developed PK model, individual post hoc estimates for fluvoxamine in plasma could be obtained for $C L, V 1, V 2$, and $Q 2$ (Table I). Interindividual variability could not be identified on the parameters $V 3$ and $Q 3$, and therefore the estimates for the population of 187 animals were used. The individual post hoc estimates were used in the current studies for each animal to estimate the fluvoxamine concentrations in plasma as input function in the brain

\section{Model for Fluvoxamine Brain Distribution}

In Fig. 2, observed fluvoxamine concentrations in ECF (a) and total brain (b) are depicted as well as the upper and lower limit of interquantile range and the median concentration versus time for a number of 2,000 datasets that were simulated from the obtained PK parameter estimates. Fluvoxamine was transported rapidly into the brain and maximal ECF and brain concentrations were observed only about 20 min later than maximal plasma fluvoxamine concentration. Since maximum concentrations in ECF and brain were observed at the same time after administration,

Table I. Mean Post Hoc Estimates for the Pharmacokinetic Parameters of Fluvoxamine in Plasma after i.v. Administration in 30 min (1, 3.7

\begin{tabular}{|c|c|c|c|c|c|c|}
\hline \multirow{2}{*}{ Study } & \multicolumn{6}{|c|}{ PK Parameter (unit) } \\
\hline & $\mathrm{CL}\left(\mathrm{ml} \cdot \mathrm{min}^{-1}\right)$ & $V 1(\mathrm{ml})$ & $V 2(\mathrm{ml})$ & $Q^{2}\left(\mathrm{ml} \mathrm{min}^{-1}\right)$ & $V 3(\mathrm{ml})$ & Q3 $\left(\mathrm{ml} \cdot \mathrm{min}^{-1}\right)$ \\
\hline Microdialysis+brain sampling & 31.6 & 321 & 949 & 33.7 & 136 & 1.0 \\
\hline Microdialysis & 36.5 & 412 & 1,255 & 39.4 & 136 & 1.0 \\
\hline Brain sampling & 28.0 & 253 & 721 & 29.4 & 136 & 1.0 \\
\hline
\end{tabular}
and $7.3 \mathrm{mg} \cdot \mathrm{kg}^{-1}$ ) Obtained by the Three-Compartment Pharmacokinetic Model

Depicted are the population mean estimates for CL, V1, V2, $Q 2, V 3$, and $Q 3$. 
0100200300
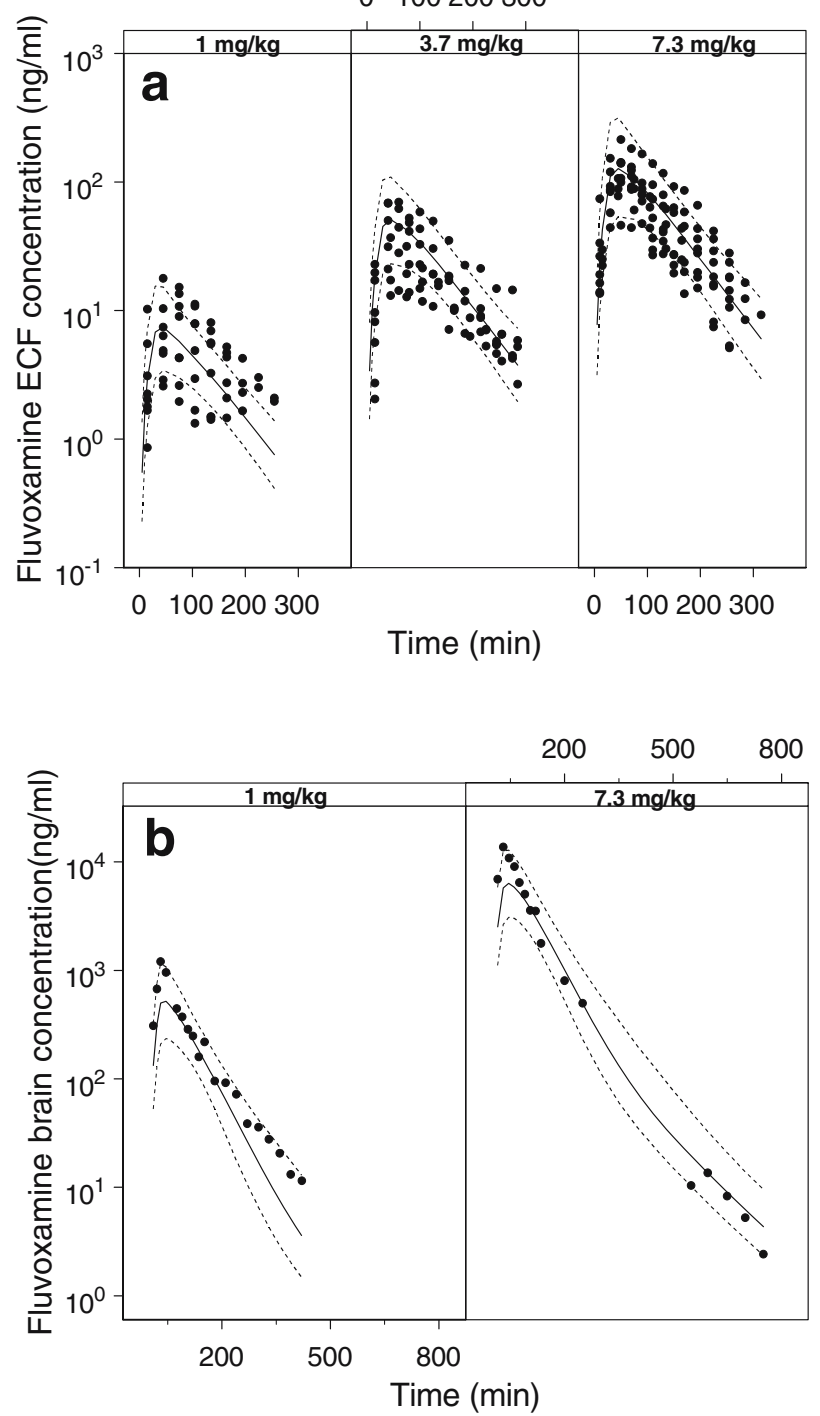

Fig. 2. Fluvoxamine concentration-time profiles in ECF (a) and total brain (b), obtained after a 30-min intravenous infusion in Wistar rats. Depicted are the observed fluvoxamine concentrations (dots), the model-simulated upper limit of the interquantile concentration range (90\%, upper dashed line), the model simulated lower limit of the interquantile concentration range $(10 \%$, lower dashed line), the median concentration (solid line) versus time. A number of 2,000 datasets were simulated from the final PK parameter estimates.

distribution between fluvoxamine in ECF and total brain was very rapid. Furthermore, fluvoxamine concentration-time curves in ECF and brain showed the same kinetic profiles without any delay or different elimination kinetics (Fig. 2).

When observed fluvoxamine concentrations were normalized for dose (normalization dose $4 \mathrm{mg} . \mathrm{kg}^{-1}$ ), nonlinearity could be observed in ECF and brain, which was not observed in plasma (Fig. 3). Specifically, with increasing fluvoxamine dose a disproportional increase in brain ECF and brain tissue concentrations relative to the plasma concentrations was observed. Therefore, the proposed PK model with saturable efflux transport from the deep brain compartment to the shallow perfusion-limited brain compartment was compared with a model without such saturable efflux transport. The proposed PK model with non-linearity in brain distribution resulted in a significantly better description of fluvoxamine concentrations in brain ECF and brain tissue compared with the linear brain model as reflected in a decrease in MVOF of 37 points. Fluvoxamine concentrations in ECF and brain could be adequately described with this model, as shown in Fig. 2. These Figures also show that the concentration-time curves of fluvoxamine in ECF and total brain follow the same kinetic profiles. Therefore, the assumption of the high rate constants between the compartments in the model was valid and the concentration-time profiles in ECF and brain were separated only by a scaling factor.

The structural parameters of the model could be simultaneously estimated with good precision (Table II). The influx rate constant in the brain $\left(k_{i n}\right)$ was $0.16 \mathrm{~min}^{-1}$ and the efflux rate from the brain by the shallow perfusionlimited brain compartment $\left(k_{\text {out }}\right)$ was $0.019 \mathrm{~min}^{-1}$.

Fluvoxamine concentration at which $50 \%$ of saturation of the active removal flux is reached $\left(C_{50}\right)$ was equal to $710 \mathrm{ng} \cdot \mathrm{ml}^{-1}$. Since measured fluvoxamine ECF concentrations ranged between 1 and 214 ng.ml ${ }^{-1}$, full saturation of the active removal flux was not reached in the current studies. The residual error for fluvoxamine concentrations in ECF and total brain $\left(\varepsilon_{1 i j}\right)$ was estimated to be equal to 0.042 and significantly lower than the estimated value by the model without incorporated saturation kinetics. Therefore, more variation could be explained by the model in which saturation of an active efflux transport was assumed, thus supporting the validity of the complex model.

The coefficients of variation (C.V.) for the ECF and brain parameters were lower than $33 \%$ for al parameters, except for $C_{50}$ and $N_{* * * \max }$ for which the coefficients of variation were 96.8 and $92.5 \%$, respectively. This can be explained by the fact that full saturation of the efflux transporter was not reached in the present investigation. Inter-individual variability (IIV) for $k_{\text {in }}$ was equal to 0.50 and for $k_{\text {out }}$ equal to 0.17 . Inter-individual variability in the other parameters could not be adequately estimated and was fixed to zero. Correlation between the variability in the estimated PK parameters was observed for $k_{\text {in }}$ and $k_{\text {out }}$ and was implemented in the model. In Fig. 4, goodness-of-fit plots for fluvoxamine concentrations in ECF of the frontal cortex and total brain are depicted. Observed ECF concentrations were in close agreement with individual predicted (a) and population predicted (b) ECF concentrations. No substantial or systemic deviation from the identity line was observed indicating adequate description of observed fluvoxamine ECF concentrations. Furthermore, no substantial or systemic deviation from the identity line was present for the observed fluvoxamine brain concentrations vs. individual (C) and population (D) predicted brain concentrations. Although each observation from total brain was collected from a different animal by destructive sampling, fluvoxamine brain concentrations could be adequately described.

\section{DISCUSSION}

The objective of this investigation was to develop a pharmacokinetic model for estimation of the time course of the fluvoxamine brain concentration in rats, to be used in future PK-PD investigations. 

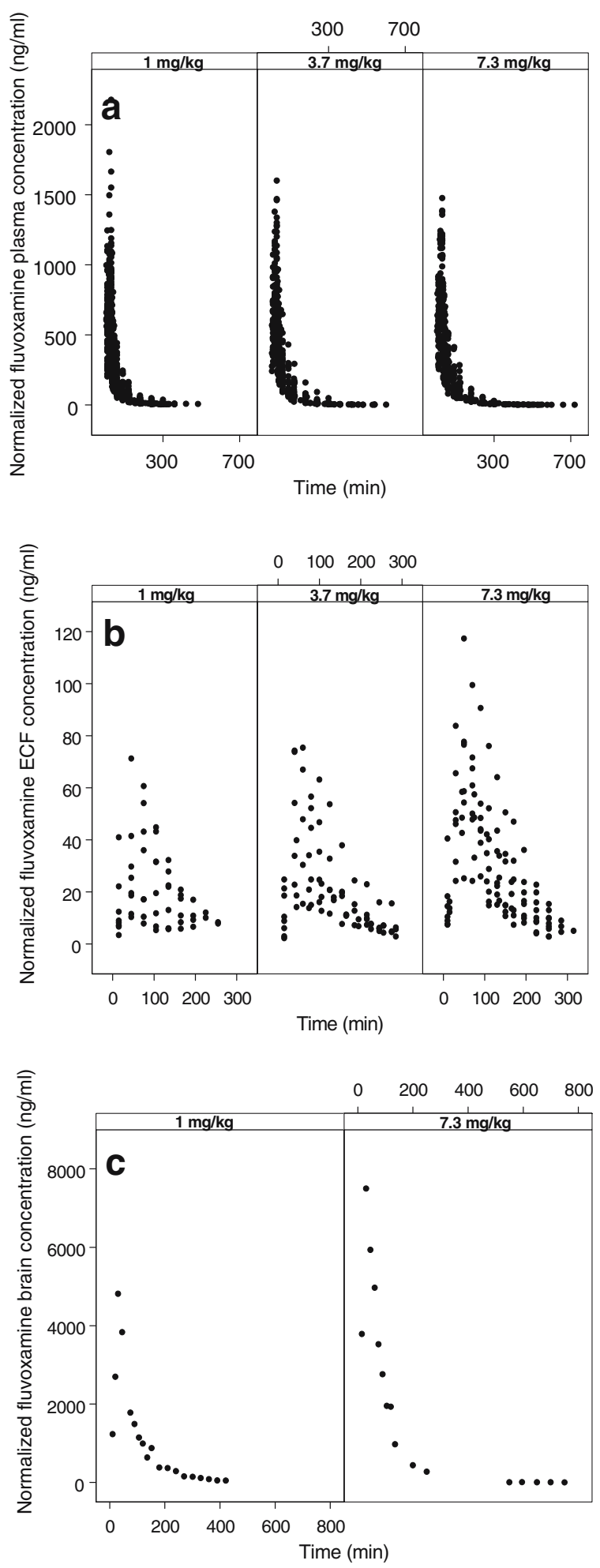

Fig. 3. Observed fluvoxamine concentration-time profiles in plasma (a), ECF (b) and total brain (c), after normalization for dose (normalization dose $4 \mathrm{mg} \cdot \mathrm{kg}^{-1}$ ) separated by fluvoxamine dose.

An important feature of the present study is that both the brain ECF concentrations (using microdialysis) as well as the brain tissue concentrations (by brain tissue sampling) were measured. By this unique approach, the time course of both the total and the free fluvoxamine concentration in brain could be estimated up to 750 min after the administration of pharmacologically relevant doses (Figs. 2 and 3). The simultaneous collection of (free) brain ECF and (total) brain tissue concentrations is conceptually important since, in theory both total and free drug concentrations can be the determinant of the pharmacodynamic response (25). Moreover the inclusion of brain concentrations enabled characterization of the brain ECF concentrations at the time points where the concentrations in microdialysate have dropped below the detection limit.

When fluvoxamine concentrations were normalized for dose, a small but distinct non-linearity was observed in the ECF and the brain concentration versus time profiles, but not in the plasma concentration versus time profiles (Fig. 3). The non-linearity in brain distribution was further supported by the results of the covariate analysis. The disproportional increase in ECF and brain concentrations reflects nonlinearity in the brain distribution. It seems unlikely that this non-linearity is caused by non-linear plasma protein binding, since fluvoxamine plasma protein binding in rats is linear over a wide concentration range. Specifically, in a separate study with the same experimental set-up and after administration of similar dosages, plasma protein binding was found to be linear with a free fraction of $2.6 \%$ (Geldof et al., unpublished observations).

In the present study, total rather free drug concentrations in plasma were measured, using serial blood sampling. In various investigations it has been demonstrated that, when using appropriate techniques, the collection of serial blood samples has minimal hemodynamic and CNS effects. An alternative would be to monitor the time course of (free) plasma concentrations by intravenous microdialysis. To date there is limited experience however with this technique in PK-PD investigations with CNS active drugs. Moreover, a theoretical disadvantage is that venous drug concentrations are obtained while the time course of the drug effect is driven by the arterial concentrations (26).

In the modeling the median time point of the microdialysis sampling interval was used as the observation time of the concentration. Thereby, the concentration of fluvoxamine was assumed to decrease in a linear fashion in each collection interval. This is justified in situations where the changes in the concentration in each collection interval are small $(27,28)$.

To overcome the adhesion of fluvoxamine to the microdialysis probe, artificial cerebrospinal fluid contained $0.5 \%$ BSA. It cannot be excluded that this affects the recovery. However, since BSA was also added in the recovery experiments, such an effect is in principle accounted for in the recovery. Furthermore, given the low BSA concentration in microdialysate an eventual effect on the recovery is likely to be minimal.

For experimental reasons, individual values of the recovery could not be obtained in 6 of the 27 rats. In these animals the mean recovery of 0.27 was used. Given the observed wide inter-animal variability in the recovery, there is a considerable uncertainty in the value of the recovery in these animals. Yet, under these circumstances the use of the mean recovery was considered the most appropriate.

The proposed PK model for description of non-linear brain distribution of fluvoxamine constitutes an extension of 
Table II. Population Pharmacokinetic Parameter Estimates of Fluvoxamine in ECF and Brain after i.v. Administration in $30 \mathrm{~min}$ $\left(1,3.7\right.$ and 7.3 mg.kg $\left.{ }^{-1}\right)$ Obtained by the Conceptual PK Model

\begin{tabular}{llcc}
\hline Parameter & Unit & Value & C.V. (\%) \\
\hline$k_{\text {in }}$ & $\min ^{-1}$ & 0.16 & 13.6 \\
$k_{\text {out }}$ & $\min ^{-1}$ & 0.019 & 8.1 \\
$N_{* * * \max }$ & ng.h & \\
$C_{50}$ & ng.ml & 30,700 & 92.5 \\
$\omega^{2} \eta_{\text {kin }}$ & - & 710 & 96.8 \\
$\omega^{2} \eta_{k \text { out }}$ & - & 0.50 & 25.7 \\
$\omega \eta_{\text {kin }} / \omega \eta_{\text {kout }}$ & - & 0.17 & 28.5 \\
$\sigma^{2} \varepsilon_{1 i j}$ & - & 0.24 & 32.9 \\
\hline
\end{tabular}

C.V.=coefficient of variation (standard error value $* 100 \%$ ) Depicted are the population mean estimates for $k_{\text {in }}, k_{\text {out }}, N_{* * * \max }$ $C_{50}, \omega^{2}, \sigma^{2}$ with corresponding coefficient of variation (C.V.)

an earlier model for the brain distribution of thiopental $(14,17)$. In this catenary two-compartment brain distribution model, a shallow perfusion-limited compartment and a deeper membrane-limited compartment are postulated, in which the distribution of drug into the deep compartment is defined by a permeability term. Interestingly, in a recent study an extended catenary biophase distribution model was found to best describe the PK-PD correlation for the EEG effect of morphine (29). The developed PK model for fluvoxamine brain distribution is an extension of the brain distribution model for thiopental by the addition of an active saturable efflux term. In this manner, it was possible to adequately estimate the time course of the total and free brain fluvoxamine concentration (Figs. 2 and 4).

In the modeling it was assumed that the values of the rate constants for diffusion between the shallow and the deep brain compartment and vice versa are identical. This was necessary since independent estimation of these rate constants was not feasible. The practice of assuming identical values of the distribution rate constants is neither unreasonable nor uncommon (8). When analyzing the data, the value of the intercompartmental distribution rate constant $k_{\text {diff }}$ rose to very high values. The parameter $k_{\text {diff }}$ could therefore be removed from the model without affecting the goodness of fit and the precision of the parameter estimates.

In the present investigation the brain distribution of fluvoxamine was studied in the pharmacologically relevant dose range of $1-7.3 \mathrm{mg} \cdot \mathrm{kg}^{-1}$. Although a distinct nonlinearity in brain distribution was detected, the precision of the parameters characterizing this non-linearity was not very high. Specifically the values for the C.V. for $C_{50}$ and $N_{* * * \max }$ were estimated as 96.8 and $92.5 \%$ respectively. This can be explained by the fact that the concentrations at which full saturation occurs lay well above the actually observed con-
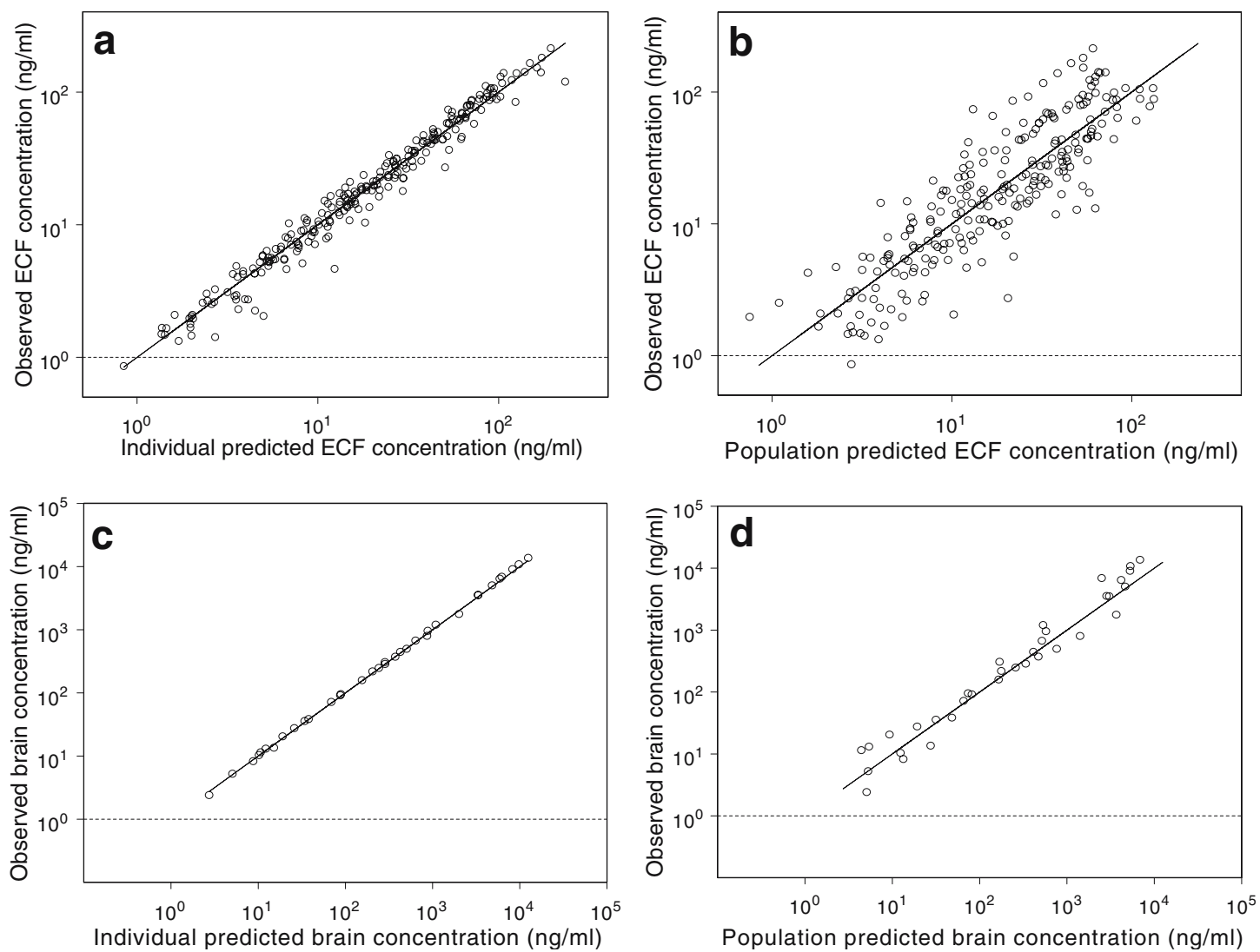

Fig. 4. Goodness-of-fit plots of fluvoxamine concentrations in ECF and total brain obtained for the physiological PK model. Depicted are scatter plots of the observed fluvoxamine ECF concentrations versus the individual model predictions (a) and population model predictions (b) and observed fluvoxamine brain concentrations versus the individual model predictions (c) and population model predictions (d). The limit of quantification $\left(1 \mathrm{ng} \cdot \mathrm{ml}^{-1}\right)$ is added for clarity (dashed line). 
centrations. Specifically, the value of the $C_{50}\left(710 \mathrm{ng} \cdot \mathrm{ml}^{-1}\right)$ lies well above the observed maximum ECF concentration of 210 ng. $\mathrm{ml}^{-1}$. Nevertheless, inclusion of a saturable function was necessary for a precise description of the brain distribution (Fig. 3). Incorporation of the saturable active efflux term in the model significantly improved the goodness of fit as reflected in a decrease of the MVOF by 37 points. Furthermore, estimates of $k_{\text {in }}$ and $k_{\text {out }}$ were not influenced by the value of parameters values for $C_{50}$ and therefore, were not dependent on a precise description of the maximal concentrations by the model at which complete saturation of the active removal flux was reached. In recent PK-PD investigation using serotonin reuptake transporter (SERT) occupancy as a pharmacodynamic endpoint, it was found that incorporation of non-linear brain distribution of fluvoxamine results in a significant improvement of the 'goodness-of-fit' (Geldof et al., unpublished observations).

In recent years much progress has been made in elucidating the mechanisms of BBB-transport of drugs. In particular, the functionality of active efflux pumps like $\mathrm{P}$-glycoprotein (Pgp) and multidrug resistance protein (MRP) appears to be an important determinant of brain concentration $(30,31)$. There are indications that fluvoxamine may indeed be a substrate for these transporters. Specifically, it was shown in vitro that fluvoxamine induces intermediate Pgp inhibition in MDR1 cells (model for human Pgp) as well as in primary porcine brain capillary endothelial cells (model for the BBB) (15), although this could not be confirmed in another study in MDR-1 cells (16). However, in this latter study the in vitro passive permeability of fluvoxamine was rather high (317 nm.s ${ }^{-1}$ ), thereby possibly obscuring the contribution of the active efflux. The role of Pgp in the brain distribution of fluvoxamine could be further explored on the basis of the interaction with specific Pgp inhibitors in microdialysis studies, as has recently been demonstrated for morphine (33).

Next to the model described in this article, an alternative model in which non-linear brain distribution was described on the basis of non-linear tissue binding to the serotonin reuptake transporter (SERT) using the concept of target-mediated disposition, (34-36) was explored. Although this model described the observed data equally well as reflected in an equal value of the goodnessof-fit parameter, the values of the parameters describing the non-linear binding in the brain were completely different from the pertinent parameters describing the binding of fluvoxamine to SERT (Geldof et al., unpublished observations). Therefore, the currently proposed model appears to be the most mechanistically plausible model to describe the observed non-linearity in the brain distribution of fluvoxamine.

In conclusion, a PK model was developed that could adequately describe observed non-linearity in fluvoxamine brain distribution. Specifically the models enables precise prediction of the time course of both the free and the total brain concentration of fluvoxamine following pharmacologically relevant doses of $1,3.7$ and $7.3 \mathrm{mg} \cdot \mathrm{kg}^{-1}$ via a $30-\mathrm{min}$ intravenous infusion in the rat. The developed PK model will be used in future mechanistic investigations on the pharmacokinetic-pharmacodynamic correlations on fluvoxamine in the rat (37).

\section{ACKNOWLEDGEMENT}

The authors would like to acknowledge the technical assistance of Willy Lorreyne and Dirk Roelant (Johnson and Johnson Pharmaceutical Research and Development) with the LC-MS/MS analyses of the plasma, microdialysate and brain samples. Susanne Bos-van Maastricht is acknowledged for her assistance with the surgeries. This project was supported by Johnson and Johnson Pharmaceutical Research and Development (a Division of Janssen Pharmaceutica N.V., Belgium).

\section{APPENDIX}

\section{Derivations of the Proposed Physiological Model for Fluvoxamine Brain Distribution}

Consider the brain to exist of two areas. The first area is a brain compartment that is in direct contact with the blood flow in which the mass balance is perfusion limited. This compartment area is defined as shallow perfusion-limited compartment (subscript SP). The second brain area is a deep brain compartment representing measured fluvoxamine concentrations in ECF (subscript $D B$ ). Fluvoxamine cannot enter this area by perfusion, but only by diffusion or active transport that could be mediated by Pgp or other transporters. The shallow perfusion-limited compartment and deep brain compartment build the total brain. The total brain concentrations were also measured in the current study.

The differential equation for the mass balance in the brain is determined by perfusion of the outer shallow perfusion-limited tissues and exchange with the deep brain compartment:

$$
\begin{gathered}
\frac{d A_{S P}}{d t}=Q_{B} C_{\text {in }}-Q_{B} C_{\text {out }}+N_{S P-D B} \\
\frac{d A_{D B}}{d t}=-N_{S P-D B}
\end{gathered}
$$

in which $A_{\mathrm{SP}}$ is the amount of fluvoxamine in shallow perfusion-limited compartment, $Q_{B}$ is the effective plasma perfusion rate, $C_{i n}$ is the fluvoxamine concentration entering the shallow perfusion-limited compartment, $C_{\text {out }}$ is the fluvoxamine concentration leaving the shallow perfusionlimited compartment, $N_{S P-D B}$ is the net mass exchange between the shallow perfusion-limited compartment and deep brain compartment and $A_{D B}$ is the amount of fluvoxamine in the deep brain compartment.

The process of mass exchange between the shallow perfusion-limited compartment and the deep brain compartment is supposed to have two components, a passive diffusive term and an active removal flux. By assuming that the latter process is saturable, the following relation can be obtained:

$$
N_{S P-D B}=-k_{d i f f}\left(C_{S P}-C_{D B}\right)+N_{\max } C_{D B} /\left(C_{50}+C_{D B}\right)
$$

in which $k_{\text {diff }}$ is the diffusion rate constant, $C_{S P}$ is the fluvoxamine concentration in the shallow perfusion-limited compartment, $C_{D B}$ is the fluvoxamine concentration in the 
deep brain compartment, $N_{\max }$ is the maximal active removal flux and $C_{50}$ is the fluvoxamine concentration in deep brain compartment at which $50 \%$ of saturation of the active removal flux is reached. Consequently the following relations are obtained:

$$
\begin{gathered}
\frac{d A_{S P}}{d t}=Q_{B} C_{i n}-Q_{B} C_{o u t}-k_{d i f f}\left(C_{S P}-C_{D B}\right) \\
+N_{\max } C_{D B} /\left(C_{50}+C_{D B}\right) \\
\frac{d A_{D B}}{d t}=k_{d i f f}\left(C_{S P}-C_{D B}\right)-N_{\max } C_{D B} /\left(C_{50}+C_{D B}\right)
\end{gathered}
$$

By assuming that the rate constants are high the following equations can be obtained:

$$
\begin{gathered}
\frac{1}{k_{\text {diff }}} \frac{d A_{D B}}{d t}=\left(C_{S P}-C_{D B}\right)-\frac{N_{\max }}{k_{\text {diff }}} C_{D B} /\left(C_{50}+C_{D B}\right) \\
0=\left(C_{S P}-C_{D B}\right)-\frac{N_{\max }}{k_{\text {diff }}} C_{D B} / C_{50}+C_{D B} \\
C_{S P}=C_{D B}+\frac{N_{\max }}{k_{\text {diff }}} C_{D B} / C_{50}+C_{D B}
\end{gathered}
$$

or

$$
A_{S P}=A_{D B} \frac{V_{S P}}{V_{D B}}+\frac{N_{\max } V_{S P}}{k_{\text {diff }}} A_{D B} / A_{50}+A_{D B}
$$

in which $V_{S P}$ is the volume in shallow perfusion-limited compartment, $V_{\mathrm{DB}}$ is the volume in the deep brain compartment and $A_{50}$ is the fluvoxamine amount in the deep brain compartment at which $50 \%$ of saturation of the active removal flux is reached.

The mass balance in the brain can be expressed in terms of one differential equation:

$$
\frac{d A_{T}}{d t}=\frac{d A_{S P}}{d t}+\frac{d A_{D B}}{d t}=Q_{B} C_{\text {in }}-Q_{B} C_{\text {out }}
$$

in which $A_{T}$ is the total amount of fluvoxamine in the brain.

Assume that the entering concentration is equal to the plasma concentration:

$$
C_{\text {in }}=C_{p}
$$

in which $C_{p}$ is the fluvoxamine concentration in plasma.

The concentration leaving the brain is determined by the partition coefficient between drug in plasma and the concentration in the shallow perfusion-limited compartment:

$$
C_{\text {out }}=C_{S P} / P
$$

in which $P$ is the partition coefficient. Accordingly:

$$
\frac{d A_{T}}{d t}=Q_{B} C_{p}-Q_{B} C_{S P} / P
$$

Dividing by brain volume results in the following equation:

$$
\frac{d C_{T}}{d t}=Q_{B} C_{p} / V_{T}-Q_{B} C_{S P} /\left(V_{T} P\right)
$$

in which $C_{T}$ is the total fluvoxamine concentration in the brain and $V_{\mathrm{T}}$ is the total brain volume.

Now, define the following constants:

$$
\begin{gathered}
k_{\text {in }}=Q_{B} / V_{T} \\
k_{\text {out }}=Q_{B} /\left(V_{T} P\right)
\end{gathered}
$$

in which $k_{\text {in }}$ is the influx rate constant in the brain and $k_{\text {out }}$ is the efflux rate constant from the brain.

As a result the following relationship is obtained:

$$
\frac{d C_{T}}{d t}=k_{\text {in }} C_{p}-k_{\text {out }} C_{S P}
$$

As a consequence we need to know the relationship between:

(1) The concentration in the deep brain compartment and total brain concentration:

$$
C_{D B}=f_{D B} C_{T}
$$

(2) The concentration in the shallow perfusion-limited compartment and total brain concentration:

$$
C_{S P}=f_{S P} C_{T}
$$

in which $f_{D B}$ is the partition coefficient for the deep brain compartment and $f_{S P}$ is the partition coefficient for the shallow perfusion-limited compartment.

Equation 37 is needed to calculate the concentration in the deep brain compartment as a function of the total concentration. Equation 38 is needed for calculating the concentration in the shallow perfusion-limited compartment and for calculating the mass balance of the total brain in Eq. 36. Substitution of Eq. 38 in Eq. 36 yields:

$$
\frac{d C_{T}}{d t}=k_{\text {in }} C_{p}-k_{\text {out }} f_{S P} C_{T}
$$

The relationships in Eqs. 37 and 38 can be found by solving Eq. 37:

$$
A_{S P}=A_{D B} \frac{V_{S P}}{V_{D B}}+\frac{N_{\text {max }} V_{S P}}{k_{\text {diff }}} A_{D B} /\left(A_{50}+A_{D B}\right)
$$

Further the relation of the total amount of fluvoxamine in the brain is known:

$$
A_{T}=A_{S P}+A_{D B}
$$

and therefore:

$$
A_{T}-A_{D B}=A_{D B} \frac{V_{S P}}{V_{D B}}+\frac{N_{\max } V_{S P}}{k_{d i f f}} A_{D B} /\left(A_{50}+A_{D B}\right)
$$

$$
A_{T}=A_{D B}\left(\frac{V_{S P}}{V_{D B}}+1\right)+\frac{N_{\max } V_{S P}}{k_{\text {diff }}} A_{D B} /\left(A_{50}+A_{D B}\right)
$$


By defining:

$$
\begin{gathered}
\alpha=\left(\frac{V_{S P}}{V_{D B}}+1\right)>1 \\
N_{* \max }=\frac{N_{\max } V_{S P}}{k_{\text {diff }}}>0
\end{gathered}
$$

the following relationship for the total fluvoxamine amount in the brain is obtained:

$$
A_{T}=A_{D B} \alpha+\frac{N_{*_{\max }} A_{D B}}{A_{50}+A_{D B}}
$$

Rearrangement results in:

$$
\alpha A_{D B}^{2}+\left(N_{*_{\max }}-A_{T}+\alpha A_{50}\right) A_{D B}-A_{T} A_{50}=0
$$

For which the solution is equal to:

with:

$$
A_{D E C F}=\frac{-b+\sqrt{b^{2}-4 a c}}{2 a}
$$

$$
\begin{gathered}
c=-A_{T} A_{50} \\
b=N_{\text {max }}-A_{T}+\alpha A_{50} \\
a=\alpha
\end{gathered}
$$

The concentration in the deep brain compartment can be calculated from the total brain concentration by:

$$
\begin{gathered}
\frac{C_{D B}}{C_{T}}=\frac{A_{D B}\left(V_{D B}+V_{S P}\right)}{A_{T} V_{D B}} \\
C_{D B}=\frac{A_{D B}\left(V_{D B}+V_{S P}\right)}{A_{T} V_{D B}} C_{T} \\
C_{D B}=f_{D B} C_{T}
\end{gathered}
$$

with the fraction:

$$
\begin{gathered}
f_{D B}=\frac{\left(V_{D B}+V_{S P}\right)}{V_{D B} A_{T}} \times \frac{-b+\sqrt{b^{2}-4 a c}}{2 a} \\
N_{* * * \max }=\frac{N_{\text {max }} V_{S P}}{k_{\text {diff }}\left(1+V_{D B}\right)}>0
\end{gathered}
$$

$$
\begin{aligned}
& f_{D B}= \\
& \frac{-N_{* * * \max }+C_{T}-C_{50}+\sqrt{\left(N_{* * * \max }-C_{T}+C_{50}\right)^{2}+4 C_{T} C_{50}}}{2 C_{T}}
\end{aligned}
$$

To calculate the concentration in the shallow perfusionlimited compartment from the total brain concentration use:

$$
C_{S P}=f_{S P} C_{T}
$$

Recall that:

$$
C_{D B}=f_{D B} C_{T}
$$

$$
A_{D B} / V_{D B}=f_{D B} A_{T} /\left(V_{D B}+V_{S P}\right)
$$

And therefore the partition coefficient for the shallow perfusion-limited compartment can be calculated by:

$$
f_{S P}=1+V_{D B} / V_{S P}\left(1-f_{D B}\right)
$$

\section{REFERENCES}

1. A. Z. Ables and O. L. Baughman III. Antidepressants: update on new agents and indications. Am. Fam. Physician 67:547-554 (2003).

2. M. Isaac. Where are we going with SSRIs?. Eur. Neuropsychopharmacol. 9 Suppl 3:S101-S106 (1999).

3. N. Bel and F. Artigas. Fluvoxamine preferentially increases extracellular 5-hydroxytryptamine in the raphe nuclei: an in vivo microdialysis study. Eur. J. Pharmacol. 229:101-103 (1992).

4. R. W. Fuller. Uptake inhibitors increase extracellular serotonin concentration measured by brain microdialysis. Life Sci. 55:163167 (1994).

5. N. Bel and F. Artigas. Reduction of serotonergic function in rat brain by tryptophan depletion: effects in control and fluvoxamine-treated rats. J. Neurochem. 67:669-676 (1996).

6. F. J. Bosker, A. A. Klompmakers, and H. G. Westenberg. Effects of single and repeated oral administration of fluvoxamine on extracellular serotonin in the median raphe nucleus and dorsal hippocampus of the rat. Neuropharmacology 34:501-508 (1995).

7. S. Benmansour, M. Cecchi, D. A. Morilak, G. A. Gerhardt, M. A. Javors, G. G. Gould, and A. Frazer. Effects of chronic antidepressant treatments on serotonin transporter function, density, and mRNA level. J. Neurosci. 19:10494-10501 (1999).

8. M. Danhof, J. DeJongh, E. C. DeLange, O. DellaPasqua, B. A Ploeger, and R. A. Voskuyl. Mechanism-based pharmacokineticpharmacodynamic modeling: biophase distribution, receptor the ory, and dynamical systems analysis. Annu. Rev. Pharmacol. Toxicol. 47:357-400 (2007).

9. M. Danhof, G. Alvan, S. G. Dahl, J. Kuhlmann, and G. Paintaud. Mechanism-based pharmacokinetic-pharmacodynamic modeling - a new classification of biomarkers. Pharm. Res. 22:14321437 (2005).

10. Biomarkers and surrogate endpoints: preferred definitions and conceptual framework. Clin. Pharmacol. Ther. 69:89-95 (2001).

11. P. Rolan. The contribution of clinical pharmacology surrogates and models to drug development-a critical appraisal. Br. $J$ Clin. Pharmacol. 44:219-225 (1997).

12. W. A. Colburn and J. W. Lee. Biomarkers, validation and pharmacokinetic-pharmacodynamic modelling. Clin. Pharmacokinet. 42:997-1022 (2003).

13. M. Hammarlund-Udenaes. The use of microdialysis in CNS drug delivery studies. Pharmacokinetic perspectives and results with analgesics and antiepileptics. Adv. Drug Deliv. Rev. 45:283-294 (2000).

14. R. N. Upton, G. L. Ludbrook, C. Grant, and D. J. Doolette. The effect of altered cerebral blood flow on the cerebral kinetics of thiopental and propofol in sheep. Anesthesiology 93:1085-1094 (2000).

15. J. Weiss, S. M. Dormann, M. Martin-Facklam, C. J. Kerpen, N. Ketabi-Kiyanvash, and W. E. Haefeli. Inhibition of P-glycoprotein 
by newer antidepressants. J. Pharmacol. Exp. Ther. 305:197-204 (2003).

16. K. M. Doan, J. E. Humphreys, L. O. Webster, S. A. Wring, L. J. Shampine, C. J. Serabjit-Singh, K. K. Adkison, and J. W. Polli. Passive permeability and P-glycoprotein-mediated efflux differentiate central nervous system (CNS) and non-CNS marketed drugs. J. Pharmacol. Exp. Ther. 303:1029-1037 (2002).

17. Y. Igari, Y. Sugiyama, S. Awazu, and M. Hanano. Comparative physiologically based pharmacokinetics of hexobarbital, phenobarbital and thiopental in the rat. J. Pharmacokinet. Biopharm. 10:53-75 (1982).

18. G. Paxinos and C. Watson. The rat brain in stereotaxic coordinates. Academic Press, 1982.

19. B. Moghaddam and B. S. Bunney. Ionic composition of microdialysis perfusing solution alters the pharmacological responsiveness and basal outflow of striatal dopamine. J. Neurochem. 53:652-654 (1989).

20. M. R. Bouw and M. Hammarlund-Udenaes. Methodological aspects of the use of a calibrator in in vivo microdialysisfurther development of the retrodialysis method. Pharm. Res. 15:1673-1679 (1998).

21. E. C. Langede, M. Danhof, A. G. Boerde, and D. D. Breimer. Methodological considerations of intracerebral microdialysis in pharmacokinetic studies on drug transport across the bloodbrain barrier. Brain Res. Brain Res. Rev. 25:27-49 (1997).

22. M. Geldof, J. Freijer, L. Beijsterveldt, P. Timmerman, A. Ahnaou, W. H. Drinkenburg, and M. Danhof. Population pharmacokinetic model of fluvoxamine in rats: utility for application in animal behavioral studies. Eur. J. Pharm. Sci. 30:45-55 (2007).

23. K. Jolling, J. J. Ruixo, A. Hemeryck, V. Piotrovskij, and T. Greway. Population pharmacokinetic analysis of pegylated human erythropoietin in rats. J. Pharm. Sci. 93:3027-3038 (2004).

24. J. A. Kuipers, F. Boer, A. Roodede, E. Olofsen, J. G. Bovill, and A. G. Burm. Modeling population pharmacokinetics of lidocaine: should cardiac output be included as a patient factor? Anesthesiology 94:566-573 (2001).

25. P. H. GraafVan der, E. A. SchaickVan, R. A. Mathot, A. P. IJzerman, and M. Danhof. Mechanism-based pharmacokineticpharmacodynamic modeling of the effects of N6-cyclopentyladenosine analogs on heart rate in rat: estimation of in vivo operational affinity and efficacy at adenosine A1 receptors. $J$. Pharmacol. Exp. Ther. 283:809-816 (1997).

26. B. Tuk, V. M. Herben, J. W. Mandema, and M. Danhof. Relevance of arteriovenous concentration differences in pharmacokinetic- pharmacodynamic modeling of midazolam. J. Pharmacol. Exp. Ther. 284:202-207 (1998).

27. P. N. Patsalos, W. T. Abed, M. S. Alavijeh, and M. T. O'Connell. The use of microdialysis for the study of drug kinetics: some methodological considerations illustrated with antipyrine in rat frontal cortex. Br. J. Pharmacol. 115:503-509 (1995).

28. L. Stahle. Pharmacokinetic estimations from microdialysis data. Eur. J. Clin. Pharmacol. 43:289-294 (1992).

29. D. Groenendaal, J. Freijer, D. DeMik, M. R. Bouw, M. Danhof, and E. C. DeLange. Influence of biophase distribution and Pglycoprotein interaction on pharmacokinetic-pharmacodynamic modelling of the effects of morphine on the EEG. Br. J. Pharmacol. Epub ahead of print (2007).

30. M. Hammarlund-Udenaes. The use of microdialysis in CNS drug delivery studies. Pharmacokinetic perspectives and results with analgesics and antiepileptics. Adv. Drug Deliv. Rev. 45:283-294 (2000).

31. J. Weiss, S. M. Dormann, M. Martin-Facklam, C. J. Kerpen, N. Ketabi-Kiyanvash, and W. E. Haefeli. Inhibition of p-glycoprotein by newer antidepressants. J. Pharmacol. Exp. Ther. 305:197204 (2003).

32. J. Weiss, S. M. Dormann, M. Martin-Facklam, C. J. Kerpen, N. Ketabi-Kiyanvash, and W. E. Haefeli. Inhibition of P-glycoprotein by newer antidepressants. J. Pharmacol. Exp. Ther. 305:197204 (2003).

33. D. Groenendaal, J. Freijer, D. DeMik, M. R. Bouw, M. Danhof, and E. C. DeLange. Population pharmacokinetic modelling of non-linear brain distribution of morphine: influence of active saturable influx and P-glycoprotein mediated efflux. Br. J. Pharmacol. Epub ahead of print (2007).

34. G. Levy. Pharmacologic target-mediated drug disposition. Clin. Pharmacol. Ther. 56:248-252 (1994).

35. A. Mager, B. Strasberg, B. Zlotikamien, C. Kaplinsky, and S. Sclarovsky. Life-threatening ventricular tachycardia as the presenting symptom of metastatic cardiac disease. Clin. Cardiol. 14:696-698 (1991).

36. D. E. Mager and W. J. Jusko. Receptor-mediated pharmacokinetic/pharmacodynamic model of interferon-beta 1a in humans. Pharm. Res. 19:1537-1543 (2002).

37. M. Geldof, J. Freijer, L. van Beijsterveldt, P. C. M. Vermote, A. A. Megens M. Danhof., Pharmacokinetic-Pharmacodynamic Modeling of the Effect of Fluvoxamine on p-Chloroamphetamineinduced behavior. Eur. J. Pharm. Sci. in press (2007). 